\title{
A ternary square-free sequence avoiding factors equivalent to abcacba
}

\author{
James Currie* \\ Department of Mathematics \& Statistics \\ University of Winnipeg \\ Winnipeg, MB \\ Canada R3B 2E9 \\ j.currie@uwinnipeg.ca
}

Submitted: Mar 10, 2016; Accepted: May 7, 2016; Published: May 27, 2016

Mathematics Subject Classification: 68R15

\begin{abstract}
We solve a problem of Petrova, finalizing the classification of letter patterns avoidable by ternary square-free words; we show that there is a ternary square-free word avoiding letter pattern $x y z x z y x$. In fact, we

- characterize all the (two-way) infinite ternary square-free words avoiding letter pattern $x y z x z y x$

- characterize the lexicographically least (one-way) infinite ternary square-free word avoiding letter pattern $x y z x z y x$

- show that the number of ternary square-free words of length $n$ avoiding letter pattern $x y z x z y x$ grows exponentially with $n$.
\end{abstract}

\section{Introduction}

A theme in combinatorics on words is pattern avoidance. A word $w$ encounters word $p$ if $f(p)$ is a factor of $w$ for some non-erasing morphism $f$. Otherwise $w$ avoids $p$. A standard question is whether there are infinitely many words over a given finite alphabet $\Sigma$, none of which encounters a given pattern $p$. Equivalently, one asks whether an $\omega$-word over $\Sigma$ avoids $p$.

The first problems of this sort were studied by Thue $[11,12]$ who showed that there are infinitely many words over $\{a, b, c\}$ which are square-free - i.e., do not encounter $x x$. He also showed that over $\{a, b\}$ there are infinitely many overlap-free words which simultaneously avoid $x x x$ and $x y x y x$. Thue also introduced a variation on pattern

\footnotetext{
*The author was supported by an NSERC Discovery Grant
} 
avoidance by asking whether one could simultaneously avoid squares $x x$ and factors from a finite set. For example, Thue showed that infinitely many words over $\{a, b, c\}$ avoid squares, and also have no factors $a b a$ or $c b c$.

In combinatorics, once an existence problem has been solved, it is natural to consider stronger questions: characterizations, enumeration problems and extremal problems. Since Thue, progressively stronger questions about pattern-avoiding sequences have been asked and answered:

- Gottschalk and Hedlund [3] characterized the doubly infinite binary words avoiding overlaps.

- How many square-free words of length $n$ are there over $\{a, b, c\}$ ? The number of such words was shown to grow exponentially by Brandenburg [2].

- Let $\mathbf{w}$ be the lexicographically least square-free $\omega$-word over $\{a, b, c\}$. As the author [1] has pointed out, the method of Shelton [8] allows one to test whether a given finite word over $\{a, b, c\}$ is a prefix of $\mathbf{w}$.

Interest in words avoiding patterns continues, and a recent paper by Petrova [7] studied letter pattern avoidance by ternary square-free words. A word $w$ over $\{1,2,3\}$ avoids the letter pattern $P \in\{x, y, z\}^{*}$ if no factor of $w$ is an image of $P$ under a bijection from $\{x, y, z\}$ to $\{1,2,3\}$. For example, to avoid the letter pattern $x y z x z y x$, a word $w$ cannot contain any of the factors 1231321, 1321231,2132312, 2312132, 3123213 and 3213123.

Petrova gives an almost complete classification of the letter patterns over $\{x, y, z\}$ which can be avoided by ternary square-free words. To do this, she uses the notion of 'codewalks', developed by Shur [9] as a generalization of the encodings introduced by Pansiot [6]. In addition to her classification, Petrova also gives upper and lower bounds on the critical exponents of ternary square-free words avoiding letter patterns $x y x z x$, $x y z x y$, and $x y x z y z$.

Regarding the particular letter pattern $x y z x z y x$, Petrova remarks at the end of her paper that '( $p$ )roving its avoidance will finalize the classification of letter patterns avoidable by ternary square-free words.'

In this note, we show that there is a ternary square-free word avoiding letter pattern xyzxzyx. In fact, we

- characterize all the (two-way) infinite ternary square-free words avoiding letter pattern $x y z x z y x$ (Theorems 1 and 2)

- characterize the lexicographically least (one-way) infinite ternary square-free word avoiding letter pattern $x y z x z y x$ (Theorem 3)

- show that the number of ternary square-free words of length $n$ avoiding letter pattern $x y z x z y x$ grows exponentially with $n$ (Theorem 4). 


\section{Preliminaries}

We will use several standard notations from combinatorics on words. An alphabet is a finite set whose elements are called letters. For an alphabet $\Sigma$, we denote by $\Sigma^{*}$, the set of all finite words over $\Sigma$; more formally, $\Sigma^{*}$ is the free semigroup over $\Sigma$, written multiplicatively, with identity element $\epsilon$. We refer to $\epsilon$ as the empty word. By a morphism, we mean a semigroup homomorphism.

If $w=u v z$, with $u, v, z \in \Sigma^{*}$, we refer to $u, v$ and $z$ as a prefix, factor, and suffix of $w$, respectively. A word $w$ over $\Sigma$ is square-free if it has no non-empty factor of the form $x x$.

By $\Sigma^{\omega}$, we denote the $\omega$-words over $\Sigma$, which are infinite to the right; more formally, an $\omega$-word w over $\Sigma$ is a function w $: \mathbb{N} \rightarrow \Sigma$, where $\mathbb{N}$ denotes the set of positive integers. By $\Sigma^{\mathbb{Z}}$ we denote the $\mathbb{Z}$-words over $\Sigma$, which are doubly infinite. Depending on context, a 'word' over $\Sigma$ may refer to a finite word, an $\omega$-word or a $\mathbb{Z}$-word.

Let $S=\{1,2,3\}, T=\{a, b, c, d\}$ and $U=\{a, c, d\}$. We put natural orders on alphabets $S, T$ and $U$ :

$$
1<2<3 \text { and } a<b<c<d .
$$

These induce lexicographic orders on words over these alphabets; the definition is recursive: if $w$ is a word and $x, y$ are letters, then $w x<w y$ if and only if $x<y$. For more background on combinatorics on words, see the books by Lothaire $[4,5]$.

Call a word over $S$ factor-good if it has no factor of the form xyzxzyx where $\{x, y, z\}=S$; i.e., the factors 1231321, 1321231, 2132312, 2312132, 3123213, 3213123 are forbidden. Call a word over $S$ good if it is square-free and factor-good. Petrova's question is whether there are infinitely many good words.

\section{$3 \quad$ Results on good words}

Theorem 1 and Theorem 2 below characterize good $\mathbb{Z}$-words. These turn out to be in 2-to-1 correspondence with square-free $\mathbb{Z}$-words over $U$.

Let $\pi$ be the morphism on $S^{*}$ generated by

$$
\pi(1)=1, \pi(2)=3, \pi(3)=2 ;
$$

thus, this morphism $\pi$ relabels 2's as 3's and vice versa.

Let $f: T^{*} \rightarrow S^{*}$ be the morphism given by

$$
f(a)=1213, f(b)=123, f(c)=1323, f(d)=1232 .
$$

Let $g: U^{*} \rightarrow T^{*}$ be the map where $g(u)$ is obtained from a word $u \in\{a, c, d\}^{*}$ by replacing each factor $a c$ of $u$ by $a b c$, each factor $d a$ of $u$ by $d b a$ and each factor $d c$ of $u$ by $d b c$.

Theorem 1. There is a $\mathbb{Z}$-word over $S$ which is good. In particular, if $\mathbf{u} \in U^{\mathbb{Z}}$ is squarefree then $f(g(\mathbf{u}))$ is good. 
Theorem 2. Let $\mathbf{w} \in S^{\mathbb{Z}}$ be good. Exactly one of the following is true:

1. There is a square-free word $\mathbf{u} \in U^{\mathbb{Z}}$ such that $\mathbf{w}=f(g(\mathbf{u}))$.

2. There is a square-free word $\mathbf{u} \in U^{\mathbb{Z}}$ such that $\mathbf{w}=\pi(f(g(\mathbf{u})))$.

We can also characterize the lexicographically least good $\omega$-word:

Theorem 3. The lexicographically least good $\omega$-word is $f(g(\mathbf{u}))$, where $\mathbf{u}$ is the lexicographically least square-free $\omega$-word over $U$.

There are 'many' finite good words, in the sense that the number of words grows exponentially with length. For each non-negative integer $n$, let $G(n)$ be the number of good words of length $n$.

Theorem 4. The number of good words of length $n$ grows exponentially with $n$. In particular, there are positive constants $A, B$ and $C>1$ such that

$$
\sum_{i=0}^{n} G(i) \geqslant A+B\left(C^{n}\right) .
$$

\section{Proof of Theorem 2}

The proof of Theorem 2 proceeds via a series of lemmas.

Lemma 5. Suppose $u \in U^{*}$. Then $f(g(u))$ is factor-good.

Lemma 6. The map $f \circ g: U^{*} \rightarrow S^{*}$ is square-free: Suppose $u \in U^{*}$ is square-free. Then so is $f(g(u))$.

Suppose that $\mathbf{w} \in \Sigma^{\mathbb{Z}}$ is good. Since $\mathbf{w}$ is square-free,

$$
\mathbf{w} \in\{12,123,1232,13,132,1323\}^{\mathbb{Z}} .
$$

These are just the square-free words over $\{1,2,3\}$ which begin with 1 and contain exactly a single 1; evidently we can partition $w$ into such blocks.

Proof.

Lemma 7. Let $w$ be a good word. Then either $|w|_{1231}=0$ or $|w|_{1321}=0$.

Proof. If the lemma is false, then either

- $w$ contains a finite factor with prefix 1231 and suffix 1321 or

- $w$ contains a factor with prefix 1321 and suffix 1231 . 
Without loss of generality up to relabeling, suppose that $w$ contains a factor with prefix 1231 and suffix 1321. Since it is good, $w$ cannot have 1231321 as a factor. Consider then a shortest factor $1231 v 1321$ of $w$; thus $|1231 v 1321|_{1231}=1$.

Exhaustively listing good words $1231 u$ with $|1231 u|_{1231}=1$, we find that there are only finitely many, and exactly three which are maximal with respect to right extension: $12312131232123,123132312131232123,12313231232123$. It follows that one of these is a right extension of $1231 v 1321$; however, none of the three has 1321 as a factor. This is a contradiction.

Interchanging 2's and 3's if necessary, suppose that $\mathbf{w}_{1321}=0$. Thus

$$
\mathbf{w} \in\{12,123,1232,13,1323\}^{\mathbb{Z}} .
$$

Lemma 8. Suppose $\mathbf{t} \in 1213\{12,123,1232,13,1323\}^{\omega}$ is good. Then

$$
\mathbf{t} \in\{1213,123,1232,1323\}^{\omega}
$$

Proof. We prove this via a series of claims:

Claim 9. Neither of 132313 and 21232 is a factor of $\mathbf{t}$.

Proof of Claim. Since $\mathbf{t} \in 1213\{12,123,1232,13,1323\}^{\omega}$, if 132313 is a factor of $\mathbf{t}$, then so is one of 1323131 and 13231323, both of which end in squares. This is impossible, since $\mathbf{t}$ is good. Similarly, if 21232 is a factor of $\mathbf{t}$, so is one of 121232 and 12321232 , both of which begin with squares.

Claim 10. Suppose that $t 12 u v$ is a factor of $\mathbf{t}$, where $t, u, v \in\{12,123,1232,13,1323\}$. Then $u=13$.

Proof of Claim. Word $u$ must be 13 or 1323; otherwise, $12 u$ begins with the square 1212 . Suppose $u=1323$. By the previous claim, v must have prefix 12. But then $2 u v$ has prefix $2132312=x y z x z y x$, where $x=2, y=1, z=3$; this is impossible. Thus $u=13$.

Claim 11. Suppose that tu13v is a factor of $\mathbf{t}$, where $t, u, v \in\{12,123,1232,13,1323\}$. Then $u=12$.

Proof of Claim. Word $u$ must end with 2; otherwise, $u 13 v$ contains the square 3131 . Thus $u$ must be 12 or 1232 . Suppose $u=1232$. By the first claim, $t$ must have suffix 3 . But then tu13 has suffix $3123213=x y z x z y x$, where $x=3, y=1, z=2$; this is impossible. Thus $u=12$.

We have proved that 12 and 13 only appear in $\mathbf{t}$ in the context 1213 . It follows that $\mathbf{t} \in\{1213,123,1232,1323\}$.

Corollary 12. Word $\mathbf{w} \in\{1213,123,1232,1323\}^{\mathbb{Z}}$. 
Figure 1: Directed graph $\mathcal{D}$

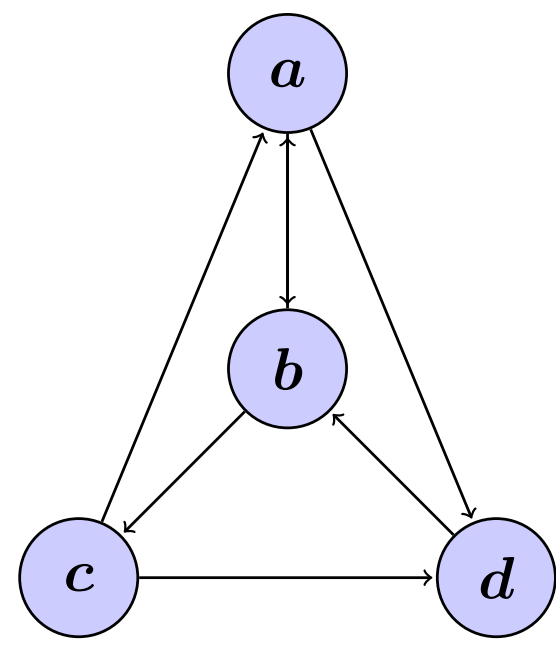

Proof. We know that $\mathbf{w} \in\{12,123,1232,13,1323\}^{\mathbb{Z}}$. If neither of 121 and 131 is a factor of $\mathbf{w}$, then $\mathbf{w}$ is concatenated from copies of $A=1323, B=1232$ and $C=123$. However, $C B$ and $A C 1$ contain squares, while $B A 12$ contains 2132312 , which cannot be a factor of a good word. This implies that $A, B$ and $C$ always occur in $\mathbf{w}$ in the cyclical order $A \rightarrow B \rightarrow C \rightarrow A$, and $\mathbf{w}$ contains the square $A B C A B C$, which is impossible. We conclude that one of 121 and 131 is a factor of $\mathbf{w}$. However, as in the proof of Claims 10 and 11, factors 12 and 13 can only occur in $\mathbf{w}$ in the context 1213, so the result follows.

By Corollary $12, f^{-1}(\mathbf{w})$ exists. Let $\mathbf{v} \in f^{-1}(\mathbf{w})$.

Lemma 13. None of $a c, a b a, b d, c b, d a$ and $d c$ is a factor of $\mathbf{v}$.

Proof. One checks that $f(a c), f(a b a), f(b d), f(c b) 1, f(d a)$ contain squares, and thus cannot be factors of $\mathbf{w}$. It follows that $a c, a b a, c b, d a$ and $d c$ are not factors of $\mathbf{v}$. On the other hand, as in the proof of the previous lemma, $f(d)=1232$ only appears in $\mathbf{w}$ in the context 123213. It follows that if $c d$ is a factor of $\mathbf{v}$, then $f(c d) 13=1323123213$ is a factor of $\mathbf{w}$. However, this has the suffix $3123213=x y z x z y x$ where $x=3, y=1, z=2$. This is impossible.

Remark 14. It follows that $\mathbf{v}$ can be walked on the directed graph $\mathcal{D}$ of Figure 1.

Let $h:\{a, b, c, d\}^{*} \rightarrow\{a, c, d\}^{*}$ be the morphism generated by $h(a)=a, h(b)=\epsilon$, $h(c)=c, h(d)=d$. Thus $h(w)$ is obtained by deleting all occurrences of $b$ in a word $w$. Suppose that $w$ is a factor of $\mathbf{v}$. If $w$ does not begin or end with $b$, then

$$
w=g(h(w)) .
$$

Let $\mathbf{u}=h(\mathbf{v}) \in U^{\mathbb{Z}}$. It follows that $\mathbf{v}=g(\mathbf{u})$, so that

$$
\mathbf{w}=f(g(\mathbf{u})) \text {. }
$$


Word $\mathbf{u}$ must be square-free; otherwise its image $\mathbf{w}$ contains a square. Thus the first alternative in Theorem 2 holds.

The other situation occurs if we decide, after Lemma 7 , that $\mathbf{w}_{1231}=0$. As we remarked at that point in our argument, this amounts to interchanging 2's and 3's, i.e., applying $\pi$. In such a case, we find that

$$
\mathbf{w}=\pi(f(g(\mathbf{u}))) .
$$

This completes the proof of Theorem 2 .

\section{Proof of Theorem 1}

Proof of Lemma 5. Let $w=f(g(u))$. Each length 7 factor of $w$ is a factor of $f\left(g\left(u^{\prime}\right)\right)$, some factor $u^{\prime} \in U^{3}$. A finite check establishes that $f\left(u^{\prime}\right)$ is factor-good for each $u \in$ $U^{3}$.

Proof of Lemma 6. Suppose for the sake of getting a contradiction, that $X X$ is a nonempty square in $w=f(v)$. If $|X| \leqslant 2$, then $X X$ is a factor of $f\left(v^{\prime}\right)$, some factor $v^{\prime}$ of $v$ with $\left|v^{\prime}\right|=2$. However, only need to consider

$$
v^{\prime} \in\{a b, a d, b a, b c, c a, c d, d b\} .
$$

(As per Remark 14, we can walk $v^{\prime}$ on $\mathcal{D}$.) In each case, we check that $f\left(v^{\prime}\right)$ is square-free. From now on, then, suppose that $|X| \geqslant 3$; in this case we can write

$$
X X=q f\left(v_{1} v_{2} \cdots v_{n-1}\right) p=q f\left(v_{n+1} v_{n+2} \cdots v_{2 n-1}\right) p,
$$

where $v_{0} v_{1} \cdots v_{n-1} v_{n} v_{n+1} v_{n+2} \cdots v_{2 n-1} v_{2 n}$ is a factor of $v, q$ is a suffix of $f\left(v_{0}\right), p$ is a prefix of $f\left(v_{2 n}\right), f\left(v_{n}\right)=p q$, and the $v_{i} \in T$. It follows that $v_{i}=v_{n+i}, 1 \leqslant i \leqslant n-1$.

If $v_{0}=v_{n}$, then $v$ contains the square $\left(v_{0} v_{1} v_{2} \cdots v_{n-1}\right)^{2}$; similarly, if $v_{n}=v_{2 n}$, then $v$ contains the square $\left(v_{1} v_{2} v_{3} \cdots v_{n}\right)^{2}$. Since $v$ is square-free, we deduce that $v_{n} \neq v_{0}, v_{2 n}$. From the condition that $f\left(v_{n}\right)$ is concatenated from a prefix of $v_{2 n}$ and a suffix of $v_{0}$, where $v_{n} \neq v_{0}, v_{2 n}$, we deduce that $v_{n}=b$.

From the definition of $g$ and the fact that $v_{n}=b$, we have $v_{n-1} v_{n} v_{1} \in\{a b c, d b a, d b c\}$. If $v_{n-1}=d$, the definition of $g$ would force $v_{n}=v_{2 n}=b$, contradicting $v_{2 n} \neq v_{n}$. We conclude that $v_{n-1} v_{n} v_{1}=a b c$. However, if $v_{1}=c$, the definition of $g$ forces $v_{n}=v_{0}=b$, contradicting $v_{0} \neq v_{n}$.

\section{$6 \quad$ Proof of Theorem 3}

Let $\mathbf{u}$ be the lexicographically least square-free $\omega$-word over $U=\{a, c, d\}$, and let $\mathbf{t}=$ $f(g(\mathbf{u}))$. It follows that $\mathbf{u}$ has prefix $a c$, so that $\mathbf{t}$ has prefix $p=f(g(a c))=f(a b c)=$ 12131231323. A finite search shows that $p$ is the lexicographically least good word of length 11. It will therefore suffice to show that $\mathbf{t}$ is the lexicographically least good $\omega$-word with prefix $p$. 
Suppose that $\mathbf{t}_{1}$ is a good $\omega$-word with prefix $p$. By Lemma 7 , it follows that $\left|\mathbf{t}_{1}\right|_{1321}=$ 0 , and from the proof of Theorem 2 , we conclude that $\mathbf{t}_{1}=f\left(g\left(\mathbf{u}_{\mathbf{1}}\right)\right)$, for some square-free word $\mathbf{u}_{\mathbf{1}}$. It remains to show that $\mathbf{u}_{\mathbf{1}}$ is lexicographically greater than or equal to $\mathbf{u}$. Suppose not.

Since $\mathbf{t}_{\mathbf{1}}$ has prefix $p$, word ac must be a prefix of $\mathbf{u}_{\mathbf{1}}$, and $\mathbf{u}, \mathbf{u}_{\mathbf{1}}$ agree on a prefix of length at least 2. Let qrs and qrt be prefixes of $\mathbf{u}_{\mathbf{1}}$ and $\mathbf{u}$, respectively, where $r, s, t \in$ $\{a, c, d\}$, and $s$ is lexicographically less than $t$.

- If $r=a$, then we cannot have $s=a$, since $\mathbf{u}_{\mathbf{1}}$ is square-free. We therefore must have $s=c$ and $t=d$. It follows that $\mathbf{t}_{\mathbf{1}}$ has prefix $f(g(q a) b c)=f(g(q a)) 1231323$, and $\mathbf{t}$ has prefix $f(g(q a) d)=f(g(q a)) 1232$, and we see that $\mathbf{t}_{\mathbf{1}}$ is lexicographically less than $\mathbf{t}$. This contradicts the minimality of $\mathbf{t}$.

- If $r=c$, then we must have $s=a$ and $t=d$. It follows that $\mathbf{t}_{\mathbf{1}}$ has prefix $f(g(q c a))=f(g(q c)) 1213$, and $\mathbf{t}$ has prefix $f(g(q c d))=f(g(q c)) 1232$, and again $\mathbf{t}_{\mathbf{1}}$ is lexicographically less than $\mathbf{t}$, giving a contradiction.

- If $r=d$, then we must have $s=a$ and $t=c$. It follows that $\mathbf{t}_{\mathbf{1}}$ has prefix $f(g(q d) b a)=f(g(q d)) 1231213$, and $\mathbf{t}$ has prefix $f(g(q d) b c)=f(g(q c)) 1231323$, and again $\mathbf{t}_{\mathbf{1}}$ is lexicographically less than $\mathbf{t}$.

We conclude that $\mathbf{u}_{\mathbf{1}}$ is lexicographically greater than or equal to $\mathbf{u}$, and $\mathbf{u}$ is the lexicographically least square-free $\omega$-word over $U$, as claimed.

\section{Proof of Theorem 4}

Let $C(n)$ be the number of length $n$ square-free words over $U$. As shown by Brandenburg [2], for $n>2, C(n) \geqslant 6\left(2^{\frac{n}{21}}\right)$. The map $f \circ g$ is injective. Since $g$ simply adds $b$ 's between some pairs of letters, $|u| \leqslant|g(u)|<2|u|$; also, $3|u| \leqslant|f(u)| \leqslant 4|u|$. Let $u \in U^{*}$ be squarefree. By the Lemmas 5 and $6, f(g(u))$ is good. Also, $3|u| \leqslant|f(g(u))|<8|u|$. We deduce that distinct square-free words over $U$ of lengths between 3 and $(n+1) / 8$ correspond to distinct good words of lengths between 9 and $n$. It follows that

$$
\sum_{i=3}^{\lfloor(n+1) / 8\rfloor} 6\left(2^{\frac{n}{22}}\right) \leqslant \sum_{i=9}^{n} G(i)
$$

and the theorem follows with $A=\sum_{i=0}^{8} G(i), B=6$ and $C=2^{\frac{1}{22}}$.

Remark 15. The growth rate of ternary square-free words is now very well understood, because of the sharp analysis by Shur [10]. One could definitely tighten the bounds of the above proof; perhaps sharp bounds could be given building on Shur's work. 


\section{References}

[1] J.-P. Allouche, J. Currie, J. Shallit, Extremal infinite overlap-free words, Electron. J. Combin., 5 (1998), \#R27.

[2] F.-J. Brandenburg, Uniformly growing $k$-th power-free homomorphisms. Theoret. Comput. Sci. 23 (1983), 69-82.

[3] W.H. Gottschalk, G.A. Hedlund, A characterization of the Morse minimal set Proc. Amer. Math. Soc., 15 (1964), 70-74.

[4] M. Lothaire, Combinatorics on Words. Cambridge University Press, Cambridge (1997).

[5] M. Lothaire, Algebraic Combinatorics on Words, Cambridge University Press, Cambridge (2002).

[6] J. J. Pansiot, A propos d'une conjecture de F. Dejean sur les répétitions dans les mots, Disc. Appl. Math. 7 (1984), 297-311.

[7] E. A. Petrova, Avoiding Letter Patterns in Ternary Square-Free Words, Electron. J. Combin. 23 (1) (2016) \#P1.18

[8] R. O. Shelton, Aperiodic words on three symbols, J. Reine Angew. Math., 321 (1981), 195-209.

[9] A. M. Shur, On ternary square-free circular words. Electron. J. Combin. 172010 , \#R140.

[10] A.M. Shur, Two-sided bounds for the growth rates of power-free languages, Developments in Language Theory 2009, Lect. Notes Comp. Sci. 5583 (2009), 466-477.

[11] A. Thue, Über unendliche Zeichenreihen, Norske Vid. Selsk. Skr. I. Mat. Nat. Kl. Christiana (1906), 1-22.

[12] A. Thue, Über die gegenseitige Lage gleicher Teile gewisser Zeichentreihen, Norske Vid. Selske Skr., I Mat. Nat. Kl. Christiania 1 (1912), 1-67. 